\title{
Análisis de Redes Sociales aplicado al estudio de los procesos de innovación agrícola
}

\author{
Mario Monge Pérez, Frank Hartwich - Instituto Internacional de Investigación \\ sobre Política Alimentaria (IFPRI) ${ }^{1}$
}

\section{Resumen}

Desde los años 70, los estudios sobre adopción de innovaciones agrícolas han estado dominados por una perspectiva según la cual la decisión de adoptar es un asunto individual, centrado en la utilidad percibida por el productor. En años recientes ha crecido el interés por comprender el papel de la interacción social en estos procesos. Poco a poco, conceptos como capital o aprendizaje social han ganado terreno entre los analistas. Sin embargo, casi ningún estudio ha utilizado el Análisis de Redes Sociales.

Con base en un conjunto de datos sobre las interacciones existentes entre productores y otros actores relevantes para la innovación en 12 micro regiones de Bolivia, se analizaron los efectos de dichas interacciones sobre la intensidad de la adopción de innovaciones, apoyándose en las herramientas provistas por los paquetes de software UCINET y NetDraw.

A nivel de micro regiones, se halló un efecto positivo y significativo de la densidad de las redes y del prestigio del promotor de las innovaciones. A nivel individual, se dio una adopción más intensa entre quienes mostraron mayor frecuencia de contacto con la agencia promotora de las innovaciones y con otros productores, mayor centralidad de grado en la red, y mayor grado de vínculos simmelianos o cohesivos (embedded ties). Además, se obtuvo evidencia significativa del efecto de la equivalencia estructural sobre los niveles de adopción. El estudio muestra además el efecto positivo de incluir variables estructurales en un modelo econométrico tradicional de adopción.

Palabras clave: Adopción de innovaciones, Redes Bi-modales, Centralidad, Vínculos simmelianos, Regresión Tobit.

\begin{abstract}
Since the 1970's, studies on adoption of agricultural innovations have been dominated by a perspective according to which adoption is an individual decision, focused on the utilities to be perceived by the farmer. In recent years there is a growing interest for understanding the role of social interaction in these processes. Social capital and social learning are concepts that have gradually received attention among scholars. But very few agricultural innovation studies have taken advantage of Social Network Analysis.

Based on a data set on interactions among farmers and other relevant actors for innovation processes in 12 micro regions of Bolivia, we analyzed the effects of those interactions on the intensity of adoption of diverse innovations, considering the tools provided by UCINET and NetDraw software packages.
\end{abstract}

1 Enviar correspondencia a: Mario Monge Pérez, mariomonge2002@yahoo.com; Frank Hartwich, frank.hartwich@gmail.com 
REDES- Revista hispana para el análisis de redes sociales

Vol.14,\#2, Junio 2006

http: // revista-redes.rediris.es

At a micro-regional level, a positive and significant effect of network density and innovation promoter's prestige on adoption was evidenced. At the level of individual farmers, adoption was more intense among those with greater frequency of contact with the promoter and with other farmers, those with a larger degree centrality, and with a larger degree of embedded or cohesive ties. Besides, significant evidence of the effects of structural equivalence on adoption levels is also presented. The positive effects of including structural variables derived from SNA into traditional econometric models are also shown.

Key words: Adoption of innovations, 2-mode networks, Centrality, Embedded ties, Tobit regression.

\section{I ntroducción}

Toda innovación es una innovación social. La innovación no ocurre 'ahí afuera' en el mundo de los objetos... la innovación puede ser propiamente comprendida sólo cuando se estudian las bases sociales de la misma (Tuomi 2002, p.5-6).

Sobre la adopción de innovaciones agrícolas existen dos grandes tradiciones que han estado bastante inconexas entre sí. Por un lado, existe una corriente de cuño sociológico que nació con los primeros estudios realizados sobre esta temática por parte de investigadores interesados en comprender las razones de la lenta difusión de los híbridos de maíz entre los productores del estado de lowa, Estados Unidos, a pesar de las evidentes ventajas de la nueva tecnología y de los esfuerzos realizados para su difusión por parte de empresas productoras y agentes de extensión ( $C f$. Ryan y Gross 1943). Para esta tradición, la difusión de innovaciones se asemeja a un proceso epidémico, en virtud del cual la decisión de adoptar de cierto productor conlleva al contagio ulterior de otros productores que están en contacto con, o son influidos por aquel. Se trata de un proceso inicialmente lento hasta que se logra alcanzar un cierto número de adoptadores capaz de acelerar el proceso decisorio de sus restantes vecinos. Finalmente el proceso se desacelera nuevamente conforme se alcanza su límite superior y restan por decidirse sólo aquellos productores más renuentes y aislados. El contagio se da básicamente mediante la interacción entre productores, en tanto que los esfuerzos de los agentes externos tienen efecto sobre la toma de decisiones de una minoría de productores, los pioneros e innovadores, quienes están más atentos a las novedades externas (Cf. Rogers 2003).

Por otra parte, entre especialistas en economía agrícola surgió pronto una tradición de impronta individualista, de acuerdo con la cual los productores toman la decisión de adoptar según las utilidades que podrían, en su criterio, derivar de tal decisión. Para ello, cada productor sopesa de forma racional los costos y beneficios esperados antes de decidir; y como existe una enorme heterogeneidad en las 
REDES- Revista hispana para el análisis de redes sociales

Vol.14,\#2, Junio 2006

http: // revista-redes.rediris.es

características individuales y las limitaciones que cada productor encara, de igual forma es de esperar una dispersión en su toma de decisiones (Cf. Griliches 1957).

A partir de los años 1970, y por diversas razones, la tradición individualista asumió un papel dominante en el estudio de la innovación agrícola, dando respaldo teórico y empírico a muchas de las políticas, programas y sistemas que se han sucedido en nuestros países a fin de promover el cambio tecnológico entre los productores y el desarrollo agrícola en general. Entre tanto, la corriente más "interaccionista" perdió interés entre los sociólogos rurales y continuó su desarrollo en otras disciplinas (Cf. Ruttan, 1996).

No obstante, en años recientes ha surgido un reconocimiento creciente de la fundamental influencia que ejercen las interacciones sociales sobre el comportamiento económico individual, el crecimiento económico, la innovación y el desarrollo rural (Cf. Goyal 2007, Fafchamps 2006, Granovetter 2005, Bebbington 1999, Manski 2000, Durston 2002, Woolcock y Narayan, 2000). Este remozado interés por las interacciones sociales se halla reflejado en la cuantiosa literatura surgida en la última década a propósito del capital social, los sistemas de innovación y el enfoque evolucionista-institucionalista en economía (Cf. Serageldin y Dasgupta 2001, Nelson y Nelson 2002, Fagerberg et al. 2004). Su impulso se ha extendido lentamente hasta el estudio de la innovación agrícola, donde cada vez son más frecuentes los estudiosos que consideran variables relacionadas con estos aspectos en sus modelos econométricos de adopción (Cf. Pomp y Burger 1995, Boahene 1995, Isham 2002, Caviglia-Harris 2003, Moser y Barrett 2006, Munshi 2005, Feder y Savastano, 2006, Moxley y Lang 2006, Grootaert y van Bastelaer 2008, Katungi et al 2008).

Llama por ello la atención el hecho de que tales estudios han sacado muy poco partido de las potencialidades del análisis de redes sociales para abordar de una forma más precisa la interacción social, limitándose básicamente a considerar el número de contactos del actor focal como Proxy de su red social. Más allá de la posible desconfianza en los enfoques y métodos provenientes de otras disciplinas, y de la poca difusión que han tenido entre economistas las metodologías del ARS y las teorías sociales que le subyacen, cabe argüir que existen obstáculos importantes a sortear para poder integrar el ARS en estudios econométricos, especialmente por las marcadas divergencias en cuanto a metodologías de muestreo y análisis. 
REDES- Revista hispana para el análisis de redes sociales

Vol.14,\#2, Junio 2006

http: // revista-redes.rediris.es

El presente estudio pretende contribuir a un mayor acercamiento entre las distintas tradiciones de análisis de la innovación agrícola mediante la realización de un estudio de adopción que integra diversos descriptores de la interacción social, derivados del ARS, como variables independientes en un modelo econométrico. EI estudio considera los efectos de los vínculos del productor con diversos actores relevantes para sus decisiones sobre el volumen o intensidad de adopción. Se pone atención particular a la interacción del productor con los agentes externos (e.g., vendedores de insumos, compradores de producto, extensionistas, etc.) que son ampliamente reconocidos como actores clave en la literatura sobre difusión de innovaciones. Se exploran distintas hipótesis derivadas de las diversas disciplinas que dan cuenta de los procesos sociales que subyacen a este tipo de decisiones.

El documento está estructurado de la siguiente forma. En la sección 2 se presenta el marco conceptual, donde se subrayan algunas de las particularidades del enfoque utilizado para este estudio y se van destacando asimismo los distintos elementos que dan forma, posteriormente, a las hipótesis de trabajo. La sección 3 describe la metodología utilizada, detallando particularmente en el procesamiento y análisis de los datos con ayuda de los métodos de ARS. La sección 4 describe los resultados. El artículo concluye con una discusión sobre las implicaciones del presente estudio de cara a la mejora de las políticas e intervenciones que pretenden dar impulso a la innovación por parte de los productores menos favorecidos, así como con algunas reflexiones al respecto de las aplicaciones y mejoras futuras al enfoque propuesto.

\section{Marco teórico}

El análisis de los distintos aspectos de la interacción social que influyen sobre las decisiones de los productores de cara a la adopción de innovaciones ha sido abordado desde muy diversas perspectivas. Para algunos economistas, las redes sociales resultan determinantes como fuentes de acceso a la información, insumos, infraestructura e instituciones requeridas para implementar la innovación (Kranton y Minehart 2001, Okten y Osili 2004, Hogset 2005, Fafchamps 2007). La mayoría de sus estudios, sin embargo, se ha concentrado en la importancia de la interacción a través del aprendizaje social, i.e., el proceso por el cual los productores aprenden de la existencia de la innovación y sus características, y se aprovechan de las experiencias de los vecinos que les han antecedido en adoptar la innovación, para actualizar sus creencias y actitudes mientras se reduce el nivel de incertidumbre que deben enfrentar de cara a la adopción (Cf. Foster y Rosenzweig 1995, Conley y Udry 2001, 2004, Munshi 2005, Abadi Ghadim et al. 2005, Yamauchi, 2007). Los 
REDES- Revista hispana para el análisis de redes sociales

Vol.14,\#2, Junio 2006

http: // revista-redes.rediris.es

modelos que consideran los efectos del aprendizaje social son capaces de predecir el fenómeno de "conformidad localizada" (i.e. la homogeneización del comportamiento a nivel local) como producto de una mayor proximidad física e interacción entre productores, observación largamente reconocida por geógrafos y sociólogos rurales. Otros estudios econométricos que han enfatizado en la importancia de la variedad y frecuencia de contacto con las fuentes de información, proponen que una mayor exposición a fuentes apropiadas de información, a través de diversos canales, debería reducir la incertidumbre subjetiva y conducir a una adopción más temprana.

Este tipo de estudios podría enriquecerse enormemente si se consideran otros efectos de la interacción social que han sido puestos de manifiesto por el análisis de redes sociales en otros campos. EI ARS se ha utilizado incesantemente desde mediados del siglo XX para el estudio de la difusión de información e innovaciones en mercadotecnia, desarrollo industrial, sociología médica y otros campos. No obstante, su aplicación al estudio de la difusión de innovaciones agrícolas ha sido mucho más limitada en cantidad y profundidad (Cf. Boahene et al. 1999, Conley y Udry 2001, Nyblom et al. 2003, Hogset 2005, Bandiera y Rasul 2006, Van den Broeck y Dercon 2007, Katungi et al. 2008). Se reseñan por ello algunos de los aprendizajes generados en otros campos, para derivar de ellos un conjunto de hipótesis que pretende ser de utilidad para futuros estudios en este ámbito.

\section{Aportes del enfoque de Redes Sociales}

Las redes sociales afectan la difusión de innovaciones a través de sus efectos sobre procesos de aprendizaje social, evaluación conjunta, influencia social y acción colectiva (Kohler et al. 2007, Hogset 2005). Los efectos del aprendizaje social se describieron más arriba. La evaluación conjunta permite a los miembros de la red reinterpretar y moderar innovaciones de riesgo, tornándolas más realistas y significativas para el contexto local. La influencia social implica los mecanismos que fuerzan al acatamiento de las normas sociales, así como los efectos de las opiniones y actitudes prevalecientes sobre las preferencias y la conducta del individuo $^{2}$. Finalmente, las redes actúan como mecanismos que ayudan a resolver las externalidades y los problemas de coordinación para la acción colectiva.

2 Degenne y Forsé (1999) indican, por ejemplo, que en comunidades rurales tradicionales, la comunicación se da a través de un red estrecha de contactos afectivos (parientes, amigos, vecinos) o normativos (instituciones y jerarquías locales) en un contexto que da preminencia a la seguridad, la seguridad y la tradición, de forma que las innovaciones deben enfrentar prejuicios desde un principio. 
REDES- Revista hispana para el análisis de redes sociales

Vol.14,\#2, Junio 2006

http: // revista-redes.rediris.es

La mayoría de los estudios de difusión realizados con un enfoque de redes sociales visualizan la difusión como un proceso de comunicación homogenizador, por el cual las actitudes y conductas individuales se ven influenciadas por la micro estructura social (Wejnert 2002). Se trata entonces de un proceso de Contagio determinado por factores de cohesión social, equivalencia estructural, popularidad, rango (acceso) y proximidad espacial, debido a los cuales los investigadores han generado distintos modelos para predecir los efectos de la estructura sobre el cambio en las actitudes entre personas ligadas por distintos tipos de relación y que ocupan ciertas posiciones en la estructura ${ }^{3}$.

- Cohesión: la interacción directa entre individuos, especialmente entre miembros de subgrupos de gran proximidad afectiva, explica en buena medida los procesos de adopción. La cohesión facilita el intercambio de conocimientos al reducir los impedimentos competitivos y motivacionales que existen para ello, en especial por el hecho de que tal transferencia es beneficiosa para el receptor pero costosa para la fuente; la presencia de vínculos densos con terceros alrededor de una relación ayuda a superar tales impedimentos (Reagans y McEvily 2003). Además, los subgrupos densos (cliques) contienen a muchos de los referentes preferidos por sus miembros individuales y generan en éstos un sentido de pertenencia que impone sobre ellos mayor presión para comportarse conforme a lo esperado. Debido a ambos efectos (comparación-competencia y presión hacia la conformidad), a mayor densidad de vínculos dentro de un grupo o subgrupo, mayor la influencia y la similitud esperable entre sus miembros. Los vínculos fuertes (homófilos) que caracterizan las relaciones cohesivas, son clave para la difusión al interior de la red (especialmente para la transmisión de conocimiento tácito, que es característico de las innovaciones técnicas), debido a su rol relevante en los procesos de influencia, comparación y

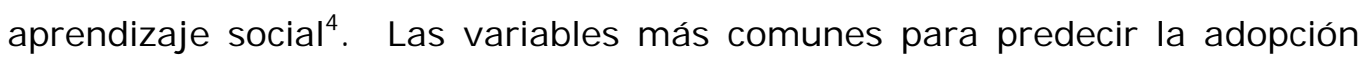
están por ello inversamente relacionadas con el tamaño de la red, y son

\footnotetext{
3 Ver por ejemplo: Burt 1978, 1980, 1987 y 2005; Erickson 1988; Friedkin 1984, 1999; Friedkin y Johnssen 1997; Marsden y Friedkin 1994; Michaelson y Contractor 1992; Mizruchi 1993; Obstfeld 2005; Reagans y McEvily 2003; Strang y Soule 1998; Valente 1996 y 2005.

${ }^{4}$ Sobre los efectos de la homofilia, autores como McPherson et al. (2001) indican que la difusión se ve impulsada entre actores con características sociales, organizacionales y estratégicas similares, dado que la gente percibe como más sencillo y relevante aprender de aquellos que se encuentran en condiciones similares. Sin embargo, la homofilia puede operar en sentido contrario, favoreciendo la resistencia a la innovación. Por ello, otros autores señalan que se requiere de un cierto nivel de heterofilia (y de vínculos débiles) para que la innovación se difunda entre distintos subgrupos, lo cual es un elemento clave en el análisis del papel de los líderes de opinión (Dagenne y Forsé 1999, Feder y Savastano 2006).
} 
REDES- Revista hispana para el análisis de redes sociales

Vol.14,\#2, Junio 2006

http: // revista-redes.rediris.es

directamente proporcionales a la densidad de la misma. La frecuencia de interacción entre los miembros refleja también ambos efectos.

- Equivalencia Estructural: Para autores como Burt (1987), la competencia entre personas de estatus similar (i.e., entre personas con grupos de referencia y posición estructural semejantes) es la fuerza motriz del contagio. Las personas que poseen vínculos con tipos similares de personas, tienden a comportarse de forma similar, aún y cuando no existan conexiones directas entre ellas. A mayor equivalencia estructural entre dos actores, mayor similitud se esperará en su conducta. De acuerdo con Wejnert (2002), la equivalencia estructural se halla determinada por factores demográficos (género, edad, raza, estado civil), sociales (educación, ocupación), y culturales (lenguaje, tradición, religión, valores y normas).

- Rango: Los vínculos débiles y heterófilos (Liu y Duff 1972, Granovetter 1973 y 1982, Hansen 1999, Levin y Cross 2004) son fundamentales durante la difusión temprana de nuevas ideas y comportamientos, debido a que ellos proveen acceso a otras partes de la red que de otra forma se hallarían desconectadas, así como vínculos a fuentes externas y no redundantes de información. Burt $(1992,2005)$ revisó la hipótesis de Granovetter sobre la "fuerza de los vínculos débiles", sugiriendo que, más que en la debilidad del vínculo, la fuerza de estos vínculos descansa en la función "puente" de estos actores que amplían el rango de la red y cubren los vacíos estructurales de la red, actuando como intermediarios de la información entre subgrupos. A causa de este papel, tales intermediarios usualmente son adoptadores tempraneros de la innovación. Además, la ventaja posicional que estos intermediarios poseen constituye su "capital social".

- Popularidad: Los miembros prominentes y bien conectados (i.e., aquellos con alta centralidad), que son usualmente líderes de opinión, generalmente adoptan pronto aquellas innovaciones que son consistentes con las normas grupales y se resisten a adoptar aquellas innovaciones que no encajan con las mismas. Al contrario, los miembros marginales de la red se ven menos afectados por los juicios del resto, por lo que con mayor probabilidad se les halla entre los primeros en adoptar las innovaciones no congruentes con los valores y normas grupales (Becker 1970, Rogers 2003). Esta línea de argumentación explica también el contagio en relaciones no recíprocas: por un lado, los miembros de menor rango podrían verse motivados a adoptar debido a su aspiración a parecerse a sus líderes; por otro, la decisión de 
REDES- Revista hispana para el análisis de redes sociales

Vol.14,\#2, Junio 2006

http: // revista-redes.rediris.es

adoptar por parte de miembros prestigiosos puede arrastrar a otros por la misma vía al variar los patrones locales de juicio.

- Proximidad Espacial: El contagio entre actores físicamente próximos es uno de los hallazgos más comunes en la literatura sobre difusión. La proximidad geográfica facilita diferentes tipos de interacción y procesos de influencia; por ello, los datos de ubicación geográfica suelen tener un buen poder de predicción de los patrones de difusión. Estos modelos suponen que los miembros de la red están distribuidos de tal forma en el espacio social que su cercanía física se corresponde con su cercanía relacional: cuanto más cerca vivan dos personas, más fuertemente vinculados deberán estar, más probable es que posean los mismos referentes $y$, por lo tanto, sería de esperar que sus actitudes y conductas sean muy parecidas. Estos modelos son, sin embargo, muy restrictivos debido a que implican que no se presentan asimetrías, intransitividad o subgrupos en las relaciones existentes en la red. Nyblom et al. (2003) representan una aplicación reciente de este tipo de modelos a la difusión de innovaciones agrícolas.

Más allá de estos modelos generales, los efectos de la red sobre las actitudes y el comportamiento innovador pueden también predecirse analizando la estructura de relaciones a distintos niveles: nodal (individual), diádico, triádico, subgrupo y red completa (Cf. Monge y Contractor 2003).

- Nivel individual: los actores con mayores niveles de centralidad (de grado) suelen ser líderes de opinión. Como se indicó más arriba, ellos usualmente adoptan más temprano que los demás las innovaciones culturalmente aceptables, y se muestran como oponentes de aquellas culturalmente inaceptables (Becker 1970). En un análisis reciente sobre las diversas medidas de centralidad y su grado de ajuste para reflejar el flujo de distintos tipos de elementos entre los actores de una red, Borgatti (2005) señala que la centralidad de grados es una buena medida de la influencia inmediata, es decir, de la probabilidad de "infección" como una función del número de actores con los cuales la productora se halla vinculada. Por otro lado, el Eigenvector de distancias geodésicas es también una buena medida para el riesgo del nodo de ser contagiado, por lo que se puede utilizar con propiedad para analizar procesos de "influencia". La diferencia entre ambas medidas es que la centralidad de grados no considera los efectos indirectos de más largo plazo. 
REDES- Revista hispana para el análisis de redes sociales

Vol.14,\#2, Junio 2006

http: // revista-redes.rediris.es

- Nivel de díadas: a este nivel, las actitudes y la similitud en el comportamiento se ven afectadas por:

- La frecuencia de interacción: a mayor frecuencia, más posibilidades de que ambas partes aprendan a interpretar las actitudes del otro de forma correcta

o La multiplicidad de interacciones: a mayor variedad de relaciones (positivas) que vinculen a dos actores, más ampliamente coincidirán ambos miembros de la díada

- La fuerza de la interacción: los vínculos más fuertes en relaciones positivas conducen a un acuerdo más fuerte entre los miembros de la díada.

- La asimetría de la interacción: en relaciones basadas en la autoridad, la asimetría puede prevenir la comparación o provocar desacuerdo, mientras que en relaciones basadas en la estima, los subordinados pueden tomar a sus superiores como modelos a imitar (Erickson 1988).

- Nivel de triada: los conceptos de balance estructural y transitividad (Wasserman y Faust 1994), han sido utilizados para predecir similitud entre actores basándose en la mera estabilidad de la estructura. La estabilidad implica que, en una estructura triádica, un no adoptador vinculado con otros dos que ya han adoptado, acabará por adoptar. Krackhardt (1998 y 1999) y Krackhardt y Kilduff (2002) señalan además que las díadas unidas por vínculos simmelianos (i.e., díadas encapsuladas en cliques de 3 personas) llegan a estar de acuerdo de forma más fuerte que los miembros de otras díadas. Extendiendo estos argumentos, podría pensarse que un actor ligado a otros dos actores que adoptan una innovación de manera intensa, adoptará también de forma intensa; y que alguien vinculado fuertemente a dos actores que han adoptado intensamente (o que promueven la innovación), y que a su vez están vinculados fuertemente entre sí, acabará por adoptar aún más intensamente que lo que podría predecirse de un análisis meramente diádico.

- Nivel de red: al compararles con estructuras más difusas, las redes centralizadas, tales como las que tienen un diseño centro-periferia, agilizan el paso de la difusión una vez que el elemento que se difunde alcanza el 
REDES- Revista hispana para el análisis de redes sociales

Vol.14,\#2, Junio 2006

http: //revista-redes.rediris.es

núcleo de líderes de opinión y otros actores de alta centralidad (Rogers, 2003) $)^{5}$.

\section{El rol de los agentes de cambio como miembros de la red}

De acuerdo con Frambach (1993), la teoría sobre difusión de innovaciones ha tomado tradicionalmente una perspectiva sesgada al lado del agente que adopta, ignorando con frecuencia la influencia del proveedor en los modelos de difusión. Incluso en los estudios que hacen énfasis en las interacciones sociales, los agentes "externos" y su posible influencia generalmente son omitidos, como si los únicos vínculos relevantes fueran aquellos existentes entre los actores locales. Sin embargo, tal omisión puede conducir a interpretaciones erróneas de los procesos ${ }^{6}$.

Parte de este sesgo puede deberse a que una de las generalizaciones básicas en la teoría clásica de la difusión de innovaciones (Rogers 2003) es que las fuentes externas de información son mayormente relevantes para diseminar información sobre la innovación en los estadios iniciales del proceso de difusión, y resultan por lo tanto relevantes sólo para persuadir a la minoría de productores (pioneros y adoptadores tempraneros), en tanto que resultan secundarias para la toma de decisiones de la mayoría de los actores, donde las interacciones entre pares adquieren un rol central. En contra de este simplismo, autores como Lin y Burt (1975) o Carey (1999) han señalado la importancia de no asimilar a los agentes de cambio con los medios masivos de comunicación, mostrando para ello el rol diferencial de los distintos interlocutores (medios masivos, extensionistas, vendedores, amigos, vecinos y otros productores) en las diversas fases del proceso de adopción.

Pero resulta claro que entre los productores y los distintos actores y agentes con quienes aquellos interactúan, se desarrolla un vínculo social capaz de incidir fuertemente sobre la toma de decisiones del productor. Se trata de una relación

\footnotetext{
${ }^{5}$ Es importante notar aquí que en los estudios sobre difusión de innovaciones agrícolas generalmente no se estudian las diferencias entre sitios distintos (o redes) distintas, por involucrar variaciones no sólo en aspectos agroecológicos sino también en otros aspectos no relacionados con el potencial agrícola, tales como diferencias en infraestructura, acceso a mercados o factores institucionales y socioculturales (Doss 2006). Los efectos locales se modelan generalmente como variables dummy, sin hipótesis alguna sobre sus posibles efectos ni medidas objetivas para capturarlos. EI ARS contribuye en este sentido con medidas objetivas y predicciones específicas sobre los efectos agregados a nivel de red (sitio).

${ }^{6}$ Por ejemplo, Van den Bulte y Lillien (2001) reanalizaron recientemente el estudio clásico de difusión de una nueva medicina entre galenos por Coleman et al. (1966), en el que se aplicó el ARS resaltando los efectos de la centralidad de los actores en la red de interacciones sociales sobre su fecha de primera prescripción de tal medicina. Su re-análisis mostró que la mayoría de los efectos originalmente atribuidos a las interacciones sociales resultaban no significativos al considerar los esfuerzos de mercadeo realizados por los distribuidores de la medicina. Por el contrario, la mayor parte de los patrones de adopción observados podía explicarse mediante las variables relacionadas con la oferta.
} 
REDES- Revista hispana para el análisis de redes sociales

Vol.14,\#2, Junio 2006

http: // revista-redes.rediris.es

asimétrica, pero los vínculos no son formales, lo cual limita la aplicabilidad de enfoques surgidos en el ámbito organizacional para explicar procesos de influencia y de transferencia de información en relaciones verticales (Cf. Sparrowe y Liden 2005, Newell y Swan 1995), donde hay más espacio para la presión coercitiva entre miembros de una jerarquía formal.

Debe indicarse, sin embargo, que tales observaciones no aplican de lleno en los estudios de adopción de innovaciones agrícolas, en los que con alguna frecuencia se ha incluido la interacción de los productores con los servicios de extensión como un factor determinante del proceso, con resultados por lo general positivos y significativos en la velocidad de difusión (Cf. Coleman 1951, Wilkening 1956, Rogers y Beal 1958, Copp et al. 1958, Polgar et al. 1963, Lin y Burt 1975, Opare et al. 1977, Feder et al. 1987, Birkhaeuser et al. 1991, Strauss et al. 1991, Hussain et al. 1994, Glendinning et al. 2001, Dinar et al. 2007).

En estos estudios, por lo general, el énfasis no recae tanto en el hecho de la interacción social, cuanto en la extensión como suplemento al capital humano del productor y como mecanismo de reducción de la incertidumbre. Por lo tanto, dicha variable se asocia regularmente con la decisión de adoptar o no, y por lo tanto, con la prontitud con que se toma la decisión inicial de hacerlo. Casi no se encuentran, por ello, estudios que hayan vinculado la intensidad de la interacción con los agentes de cambio con la intensidad de la adopción.

No obstante, la promoción de innovaciones entre productores pobres de los países en desarrollo por parte de proyectos y extensionistas va usualmente acompañada de una diversidad de incentivos que buscan reducir el riesgo a enfrentar por el productor y a paliar sus limitadas capacidades de absorción y de inversión, tales como la provisión subsidiada de los insumos, infraestructura y asesoría técnica requeridas, el apoyo necesario en la comercialización del producto para asegurar ganancias al hogar mientras se incuban sus capacidades empresariales, etc.). El despliegue de tales mecanismos tiene el efecto de rendir curvas de difusión más inclinadas, que alcanzan sus límites superiores en plazos más cortos. Y su aplicación es aún más intensiva por parte de aquellos proyectos urgidos por cumplir con las metas cuantitativas y los plazos permitidos por los organismos financieros.

Por tales motivos, es razonable argüir que los agentes de cambio tienen un papel mucho más influyente sobre las tasas de adopción de los pequeños productores que lo que usualmente se reconoce en la literatura. $Y$ tal influencia estará mediada forzosamente por el grado de interacción que surja entre dichos agentes y los 
REDES- Revista hispana para el análisis de redes sociales

Vol.14,\#2, Junio 2006

http: //revista-redes.rediris.es

productores, razón por la cual, es fundamental considerar a tales actores como parte de la red social en que los productores agrícolas se desempeñan.

\section{Hipótesis a analizar}

Con base en los aportes anteriores se formuló el siguiente conjunto de hipótesis sobre los efectos de la interacción social sobre la adopción de innovaciones agrícolas:

\begin{tabular}{|c|c|c|}
\hline Nivel & Hipótesis & Operacionalización de la hipótesis \\
\hline \multirow[t]{3}{*}{$\begin{array}{l}\text { Regional } \\
\text { (red) }\end{array}$} & $\begin{array}{l}\text { 1. Regiones con mayor } \\
\text { interacción social presentan } \\
\text { tasas promedio de adopción } \\
\text { más intensas. }\end{array}$ & $\begin{array}{l}\text { Redes con mayor densidad de vínculos presentan } \\
\text { mayores tasas promedio de adopción }\end{array}$ \\
\hline & $\begin{array}{l}\text { 2. Regiones en donde la } \\
\text { interacción está más } \\
\text { concentrada alrededor de } \\
\text { pocos actores centrales } \\
\text { presentan una difusión más } \\
\text { rápida }\end{array}$ & $\begin{array}{l}\text { A mayor centralización de la red, menor será el } \\
\text { lapso transcurrido desde el momento en que los } \\
\text { productores cobran conciencia de la innovación } \\
\text { hasta su adopción }\end{array}$ \\
\hline & $\begin{array}{l}\text { 3. Regiones en que el } \\
\text { promotor tiene más prestigio } \\
\text { entre los agentes de cambio } \\
\text { adoptan más rápida e } \\
\text { intensamente }\end{array}$ & $\begin{array}{l}\text { A mayor centralidad de grados del promotor del } \\
\text { conjunto de innovaciones en la red de agentes de } \\
\text { influencia social, mayores tasas promedio y menor } \\
\text { el lapso promedio requerido para su adopción }\end{array}$ \\
\hline \multirow[t]{8}{*}{ Individual } & $\begin{array}{l}\text { 3. Productores }{ }^{7} \text { con una } \\
\text { mayor interacción con otros } \\
\text { actores adoptan de forma } \\
\text { más rápida e intensa }\end{array}$ & $\begin{array}{l}\text { Según sea mayor el número de actores con quienes } \\
\text { un productor interactúa de forma frecuente o muy } \\
\text { frecuente al respecto de la innovación (i.e., a } \\
\text { mayor centralidad de grados en la red que muestra } \\
\text { sólo las interacciones frecuentes y muy frecuentes } \\
\text { entre actores), más rápida e intensa será su } \\
\text { adopción. }\end{array}$ \\
\hline & \multirow{6}{*}{$\begin{array}{l}\text { 4. Productores expuestos a un } \\
\text { esfuerzo persuasivo más } \\
\text { fuerte por parte de otros } \\
\text { actores de la red, adoptan en } \\
\text { un lapso menor y de forma } \\
\text { intensa. }\end{array}$} & La adopción es más rápida e intensa: \\
\hline & & $\begin{array}{l}\text { a) A mayor interacción del productor con el } \\
\text { promotor principal de la innovación }\end{array}$ \\
\hline & & $\begin{array}{l}\text { b) A mayor interacción del productor con otros } \\
\text { agentes técnicos }\end{array}$ \\
\hline & & $\begin{array}{l}\text { c) A mayor interacción del productor con agentes } \\
\text { del mercado (vendedores de insumos, compradores } \\
\text { de producto, transportistas) }\end{array}$ \\
\hline & & $\begin{array}{l}\text { d) A menor interacción del productor con otros } \\
\text { productores }\end{array}$ \\
\hline & & $\begin{array}{l}\text { e) A mayor interacción del productor con otros } \\
\text { actores que a su vez están fuertemente vinculados } \\
\text { con el promotor principal de la innovación (i.e., a } \\
\text { mayor índice de vínculos simmelianos) }\end{array}$ \\
\hline & $\begin{array}{l}\text { 5. El nivel de adopción de los } \\
\text { productores está determinado } \\
\text { por aquél de sus referentes } \\
\text { locales }\end{array}$ & $\begin{array}{l}\text { A mayor equivalencia estructural entre dos } \\
\text { productores, mayor similitud habrá en sus niveles } \\
\text { de adopción }\end{array}$ \\
\hline
\end{tabular}

Tabla 1. Conjunto de hipótesis a analizar en el presente estudio.

\footnotetext{
7 Para efectos de simplificación, en esta tabla, lo mismo que en el resto del documento, se hace referencia a "productores" en donde cabría indicar más correctamente "productoras y productores"
} 
REDES- Revista hispana para el análisis de redes sociales

Vol.14,\#2, Junio 2006

http: // revista-redes.rediris.es

\section{Metodología}

\section{Sitio, participantes y tipo de información recabada}

Los datos para este estudio fueron recabados entre los meses de Agosto y Diciembre de 2005, como parte de un estudio del Instituto Internacional de Investigación sobre Política Alimentaria (IFPRI) realizado con la colaboración del Sistema Boliviano de Tecnología Agropecuaria (SIBTA), las Fundaciones Trópico Húmedo y Valles, y PROINPA (una organización dedicada a la investigación y promoción de productos andinos).

Este estudio se diseñó originalmente como cualquier otra investigación de corte econométrico sobre la adopción de innovaciones, es decir, partiendo de un muestreo estratificado y aleatorio al interior de cada estrato, y recabando datos sobre el grado de uso de las innovaciones promovidas y de sus determinantes, básicamente factores relacionados con la percepción del productor sobre las utilidades que podría obtener de la innovación, y datos sociodemográficos y sobre su dotación con recursos que son determinantes de su capacidad de absorción de innovaciones.

El estudio se realizó con productores de 3 actividades agrícolas (Acuicultura, Maní y Quinua) en 3 distintas regiones de Bolivia: Trópico Húmedo, Valles y Altiplano. Por cada actividad se seleccionaron 4 distintas micro regiones de producción, y en cada una de ellas se entrevistó a un total de 30 productores, considerando al menos 5 no adoptadores en cada micro región, para una muestra conjunta de 120 productores por actividad y 360 productores en total (60 de ellos no adoptadores).

Además, dado el interés explícito de abordar el tema de las interacciones sociales, el instrumento de consulta incluyó un conjunto detallado de preguntas sobre los vínculos de cada productor entrevistado con otros actores. A cada productor se le indagó sobre su interacción con los distintos "Agentes de Influencia Social”, una categoría ad hoc compuesta por Agentes de Cambio (externos a la comunidad y que en el presente caso incluyó a extensionistas e investigadores del sector público, técnicos de proyectos y ONG, vendedores de insumos, compradores de producto y transportistas) y otros actores locales (categorizados como vecinos, parientes, organizaciones de productores y gobiernos o autoridades locales). Es importante aclarar que las únicas categorías que se abrieron para que el productor detallara sobre sus vínculos con agentes específicos, fueron las "organizaciones de investigación de gobierno" y las "ONG y proyectos". El resto de actores se trató como categorías agregadas sobre las que el entrevistado debía brindar una impresión global. 
REDES- Revista hispana para el análisis de redes sociales

Vol.14,\#2, Junio 2006

http: // revista-redes.rediris.es

Este tipo de datos corresponde a redes de afiliación o bi-modales, en donde los "actores" (i.e., los productores) reportan sobre su afiliación a los distintos "eventos" (i.e., los agentes de influencia social). La relación de interés para este estudio fue la del intercambio de información entre los actores al respecto de la innovación, valorándose de acuerdo a la frecuencia de tal interacción.

Una vez que se completó la recolección de datos entre los productores, se consolidó una lista de los agentes de influencia social específicamente mencionados en cada micro región, para entrevistar posteriormente a un representante de cada una de ellas con el fin de levantar información sobre los vínculos de su organización con los demás agentes ("eventos"), en cuanto a la provisión de información a los productores sobre las innovaciones de interés. Se utilizó para ello un instrumento similar cuyos datos permitieron contar con una matriz cuadrada de las interacciones entre los agentes de influencia social en cada micro región.

\section{Estimación de variables y análisis de datos}

La tabla 2 resume las variables utilizadas en el presente estudio.

\begin{tabular}{|c|c|c|}
\hline Variable & Descripción & Tipo \\
\hline \multicolumn{3}{|l|}{ Dependientes: } \\
\hline INTENSIDAD & $\begin{array}{l}\text { Porcentaje promedio de adopción de los componentes del } \\
\text { conjunto de innovaciones promovido en cada micro región. }\end{array}$ & Continua (\%) \\
\hline I NTENSREG & Promedio de intensidades reportadas en cada micro región & Continua (\%) \\
\hline LAPSO & $\begin{array}{l}\text { Lapso desde el momento en que se conoce del set de } \\
\text { innovaciones hasta adoptar alguno de sus componentes }\end{array}$ & Continua (años) \\
\hline LAPSOREG & Promedio de los lapsos reportados en cada micro región & Continua (años) \\
\hline \multicolumn{3}{|l|}{ Independientes: } \\
\hline \multicolumn{3}{|l|}{ a) Relacionales } \\
\hline DENSIDAD & Densidad de la red de cada micro región & Continua (índice) \\
\hline CENTRAZN & Grado de centralización de la red de cada micro región & Continua (índice) \\
\hline PRESTIGIO & Centralidad del promotor en red de agentes de cambio & Continua (índice) \\
\hline CENTRALIDAD & Centralidad de grados de cada productor & Continua (índice) \\
\hline PROMOTOR & $\begin{array}{l}\text { Frecuencia de interacción del productor con el promotor } \\
\text { principal del conjunto de innovaciones }\end{array}$ & Ordinal \\
\hline OTROSTEC & $\begin{array}{l}\text { Frecuencia de interacción con otros agentes técnicos } \\
\text { (investigadores, extensionistas, proyectos y ONG) }\end{array}$ & Ordinal \\
\hline OTROSMER & $\begin{array}{l}\text { Frecuencia de interacción con agentes ligados al mercado } \\
\text { (vendedores de insumos, compradores y transportistas) }\end{array}$ & Ordinal \\
\hline OTROSPRO & $\begin{array}{l}\text { Frecuencia de interacción con otros productores (vecinos, } \\
\text { familiares) }\end{array}$ & Ordinal \\
\hline SIMMEL & $\begin{array}{l}\text { Grado de cohesión de los vínculos del productor con otros } \\
\text { agentes de cambio ligados fuertemente al promotor }\end{array}$ & Continua (índice) \\
\hline EQUIVEST & Grado de equivalencia estructural entre pares de actores & Dummy \\
\hline \multicolumn{3}{|l|}{ b) Otras } \\
\hline DISTANC & Distancia al mercado en que comercializan el producto & Continua $(\mathrm{Km})$. \\
\hline DISTANREG & Distancia promedio al mercado en cada micro región & Continua $(\mathrm{Km})$. \\
\hline CONSUMO & Porción del producto que se destina al autoconsumo & Continua (\%) \\
\hline EDUCA & Nivel educativo del jefe de familia & Ordinal \\
\hline PROPEXPE & Propensión a la experimentación & Ordinal \\
\hline PRODUCC & Aumento esperado en producción gracias a la innovación & Ordinal \\
\hline
\end{tabular}

Tabla 2. Variables utilizadas en el estudio. 
REDES- Revista hispana para el análisis de redes sociales

Vol.14,\#2, Junio 2006

http://revista-redes.rediris.es

Se describe a continuación la forma de estimación de aquellas variables en que se consideran importantes algunos detalles.

En el caso de la INTENSIDAD de la adopción, para cada productor se estimó un promedio de acuerdo con el siguiente procedimiento: Como diversas innovaciones fueron promovidas a la vez en cada micro región, cada una fue considerada como componente del conjunto de innovaciones que era posible adoptar para el productor. La intensidad de adopción de cada componente fue estimada por el entrevistador en una escala porcentual, de acuerdo con el grado máximo posible de uso (e.g. área sembrada, producción procesada, etc.). La INTENSIDAD corresponde entonces al promedio de los grados de utilización de los distintos componentes promovidos.

Para estimar la DENSIDAD de la red, primero se obtuvo la socio-matriz del gráfico bi-partito a partir de cada red bi-modal. Luego se dicotomizó tales datos estableciendo el límite de inclusión por encima de 3 en la escala Likert, de forma que sólo se considerase como existentes aquellos vínculos con quienes los productores indicaron interactuar de forma frecuente o muy frecuente. Una vez transformados los datos de esta forma, la densidad de cada red micro-regional se estimó a través de UCINET (Borgatti et al. 2002).

La CENTRAZN se estima comúnmente con base en la fórmula propuesta por Freeman, $\Sigma\left[c_{*}-c_{i}\right] / \max \Sigma\left[c_{*}-c_{i}\right]$, que corresponde a sumar las diferencias entre $c_{*}$, i.e. el nivel de centralidad del actor que tuvo el índice más alto, y $c_{i}$, el nivel de cada uno de los demás actores, normalizadas de acuerdo al máximo nivel de centralización posible ( $\max \Sigma\left[c_{*}-c_{i}\right]$ ), que es el del gráfico en forma de estrella. En el presente caso la estimación se hizo de acuerdo con el procedimiento sugerido por Everett y Borgatti (2005) para extender las medidas de centralización a las redes bi-modales. En este estudio se utilizó básicamente la centralidad de grados de cada productor (CENTRALID), que corresponde al conteo vínculos del productor con los distintos "eventos" (agentes de influencia social) normalizado por el número máximo posible de eventos al que el productor podría haber estado vinculado. Para estimar la centralización también fue necesario estimar la centralidad de grados de cada evento, i.e., el conteo de actores que dijeron estar afiliados a un evento, normalizado según el máximo número de actores que el evento pudo haber abarcado. Con estos datos se identifica el actor de mayor centralidad en toda la red (ya sea "actor" o "evento") y se le utiliza para estimar el dividendo en la fórmula de Freeman. El divisor, a su vez, se estima de acuerdo con la siguiente fórmula propuesta por los autores citados para estimar el máximo nivel de centralización posible en redes bi-modales: $\left(n_{0}-n_{i}-n_{0}+1\right)\left(n_{i}+n_{0}\right) /\left(n_{i} n_{0}\right)$, en 
REDES- Revista hispana para el análisis de redes sociales

Vol.14,\#2, Junio 2006

http: // revista-redes.rediris.es

donde $n_{o}$ es el tamaño del set de nodos al que pertenece aquél con el mayor grado de centralidad $\left(C_{*}\right)$, y que podría ser tanto el conjunto de actores como el set de eventos, y $n_{i}$ es el set complementario.

La variable PRESTIGIO corresponde a la centralidad de grado de entrada del promotor de las innovaciones en la red de interacción entre los distintos agentes de influencia social señalados por los productores.

En el caso de las variables PROMOTOR, OTROSTEC, OTROSMER y OTROSPRO, la frecuencia de la interacción se valoró de acuerdo con la siguiente escala Likert: $5=$ muy frecuente, $4=$ frecuente, $3=$ intermedia, $2=$ infrecuente, $1=$ muy infrecuente.

SIMMEL: Para estimar este índice se utilizó el siguiente procedimiento. Primero, a partir de la red de afiliación obtenida para cada micro región, se generó una nueva red de afiliación modificada en la que se eliminó la columna correspondiente al promotor principal de la innovación en dicha localidad. La matriz así generada se multiplicó luego por una nueva matriz consistente únicamente en una columna que contenía los datos de frecuencia de interacción entre el promotor y cada uno de los demás agentes de influencia social que figuran como columnas o "eventos" en la red de afiliación modificada ${ }^{8}$. Con este procedimiento se obtuvo entonces una única columna de índices que muestra el grado en que los vínculos de cada productor con todos los otros agentes de la red distintos del promotor principal de la innovación se hallan "encapsulados" y por ello sesgados a favorecer más la innovación debido a la existencia de un vínculo fuerte entre esos agentes y el promotor principal de la innovación. No se trata en este caso de una triada, sino de díadas "simmelianas" (embedded ties) o cohesivas, en la forma descrita por Krackhardt y Kilduff (2002) y Reagans y McEvily (2003).

Para estimar el grado de equivalencia estructural, EQUIVEST, entre los distintos productores, se utilizó el siguiente procedimiento. Primero, las redes bi-modales de cada micro región fueron dicotomizadas para considerar sólo aquellos vínculos frecuentes y muy frecuentes. Luego, las redes bi-modales binarizadas fueron transformadas en matrices de 1 modo de las redes de afiliación, interesándonos básicamente la que muestra a los productores en filas y columnas, y cuyas celdas representan el número de "eventos" en que cada par de productores coincide (i.e., el número de agentes de influencia social de la micro región con los que ambos

\footnotetext{
${ }^{8}$ Estos datos, según se indicó más arriba, se obtuvieron a partir de las redes de interacción entre los agentes de influencia social, que se generaron a partir de entrevistas con todos los agentes enumerados por los productores de cada microrregión.
} 
REDES- Revista hispana para el análisis de redes sociales

Vol.14,\#2, Junio 2006

http: // revista-redes.rediris.es

productores coinciden en estar vinculados de manera frecuente o muy frecuente). Luego se estimó el grado de similitud entre cada par de actores utilizando el coeficiente de J accard ${ }^{9}$, y luego se obtuvo el grado de correlación entre la matriz de dichos coeficientes de Jaccard y otra matriz, que se generó a través de UCINET, que contenía el valor absoluto de las diferencias entre las intensidad de adopción de ambos miembros de cada díada.

Las variables EDUCA, PROPEXPE y PRODUCC, de tipo ordinal, fueron evaluadas de acuerdo con las siguientes escalas Likert: EDUCA, desde $5=$ Muy alta a $1=$ Rudimentaria; PROPEXPE, desde $5=$ le gusta mucho experimentar, hasta $1=$ no le gusta para nada; y PRODUCC, desde $5=$ muy alto, hasta $1=$ insignificante.

Para probar las distintas hipótesis indicadas anteriormente, se utilizaron las herramientas de correlación y regresión provistas por UCINET para Windows (Borgatti et al. 2002). Como se trata de datos relacionales, no independientes, se utilizaron procedimientos alternativos como pruebas de permutación o pruebas de correlación de matrices mediante procedimientos de asignación cuadrática (QAP) según lo indicado en cada caso (Cf. Krackhardt 1987 y 1988; Hanneman y Riddle 2005). Por último, las distintas variables que se consideraron como determinantes significativos de la adopción a nivel individual se combinan en sendos modelos econométricos Tobit $^{10}$ en los que se incluyen/excluyen variables relativas a la estructura de sus interacciones sociales, a fin de comparar el efecto de la inclusión de este tipo de variables.

\section{Resultados}

\section{Efectos a nivel de red (micro región)}

La tabla 3 presenta los datos obtenidos a nivel de cada micro región, para la prueba de hipótesis con respecto a los efectos de la estructura de la red sobre la intensidad y prontitud promedio de adopción de las innovaciones promovidas.

\footnotetext{
${ }^{9}$ De acuerdo con Hanneman y Riddle (2005), en redes de baja densidad, las medidas de similitud tales como "matches", "correlación" y "distancia" son inconvenientes debido a la poca variedad que muestran entre actores. El coeficiente de J accard es una buena opción debido a que calcula en número de veces en que ambos actores en una diada reportan un vínculo con el mismo evento, como porcentaje del número total de vínculos reportados, ignorando los casos en que ninguno de los actores está vinculado a ese evento.

${ }^{10}$ Este tipo de modelo es muy utilizado para regresiones en las que se tiene una variable censurada o truncada. El caso de la intensidad de innovación es uno clásico de variables censuradas, dado que se espera una cierta concentración de observaciones cuya intensidad de adopción es cero (los no adoptadores). En este tipo de casos, una regresión lineal simple: por ejemplo, puede predecir niveles de adopción negativos (Greene, 2003).
} 
REDES- Revista hispana para el análisis de redes sociales

Vol.14,\#2, Junio 2006

http: // revista-redes.rediris.es

La intensidad promedio de adopción entre las 12 micro regiones estudiadas fue de un $53.5 \%$, con un rango entre 27.4 y $72.5 \%$; el lapso promedio transcurrido desde el momento en que el productor conoció del conjunto de innovaciones propuesto hasta que decidió adoptar al menos uno de sus componentes, fue de 1.34 años (rango: 0.27 - 4.4 años). La densidad promedio de estas 12 redes fue de $33.7 \%$ (rango: 11.8 - 44.2\%); la centralización promedio fue del 58\% (rango: 38.5 $76.4 \%$ ), la distancia promedio al mercado fue de $26.5 \mathrm{Km}$. (rango: 6.3 - $74.8 \mathrm{Km}$.) y el índice promedio de prestigio del promotor de las innovaciones fue de 24.6 (rango: 5.9 - 45.8).

\begin{tabular}{lcccccccc}
\hline \multicolumn{1}{c}{ Variable } & Promedio & Desv.Est. & 1 & 2 & 3 & 4 & 5 & 6 \\
\hline 1. INTENSREG & 53.52 & 11.41 & - & & & & & \\
2. LAPSOREG & 1.34 & 1.30 & .045 & - & & & & \\
3. DENSIDAD & 33.70 & 9.78 & $.711^{* * *}$ & -.198 & - & & & \\
4. CENTRAZN & 58.06 & 11.41 & -.146 & .337 & -.519 & - & & \\
5. PRESTIGIO & 24.57 & 11.53 & .120 & $-.778^{* * *}$ & .320 & -.156 & - & \\
6. DISTREG & 26.50 & 19.69 & -.353 & .390 & -.406 & .489 & -.379 & - \\
\hline
\end{tabular}

$* * * p<.01$

Tabla 3. Promedios, desviaciones estándar y correlaciones entre las variables de nivel micro regional.

Los coeficientes de correlación obtenidos muestran una asociación positiva y significativa entre la densidad de la red y la intensidad promedio de adopción, así como negativa y significativa entre el índice de prestigio del promotor de las innovaciones (en la red de agentes de influencia social) y el lapso promedio requerido en la región para adoptar. Se observa también correlaciones altas y negativas (aunque no significativas) entre la densidad de la red y tanto la centralización de la misma como la distancia promedio a los mercados. También hay una correlación alta y positiva (aunque no significativa) entre la centralización de la red y la distancia a los mercados. En todos los casos, los signos son conforme a lo esperado: redes donde los miembros están geográficamente más alejados se corresponden con aquellas en que hay menos conexiones y una mayor dependencia alrededor de un pequeño grupo de actores más activo.

Al combinar las variables anteriores en un modelo de regresión lineal simple para cada una de ambas variables dependientes, se obtuvieron los resultados que se resumen en la tabla 4. Aún con el reducido número de redes a comparar en esta parte del estudio, las regresiones anteriores presentan evidencia estadísticamente significativa para apoyar la hipótesis 1 y parcialmente la hipótesis 3 (i.e., el prestigio del promotor afecta la prontitud con que las innovaciones son adoptadas, pero no la intensidad de tal adopción). En el caso de la hipótesis 2, los resultados 
REDES- Revista hispana para el análisis de redes sociales

Vol.14,\#2, Junio 2006

http: // revista-redes.rediris.es

obtenidos no fueron significativos e incluso el signo mismo de la relación es contrario a lo estipulado a la hipótesis.

\begin{tabular}{lcc}
\hline Variable & $\begin{array}{c}\text { Modelo } 1 \\
(\text { INTENSREG) }\end{array}$ & $\begin{array}{c}\text { Modelo 2 } \\
(\text { LAPSOREG) }\end{array}$ \\
\hline Constante & 7.608 & $0.621(2.234)$ \\
DENSIDAD & $(21.163)$ & \\
& $1.024 * *$ & $0.031(0.034)$ \\
CENTRAZN & $0.326)$ & \\
PRESTIGIO & $-0.199(0.251)$ & $-0.089 * *$ \\
& & $0.031(0.026)$ \\
DISTREG & $-0.159(0.163)$ & $0.002(0.017)$ \\
$R^{2}$ & 0.636 & 0.689 \\
$R^{2}$ ajustado & 0.429 & 0.511 \\
\hline
\end{tabular}

Error estándar entre paréntesis; $* * p<.05$

Tabla 4. Coeficientes de regresión lineal (OLS) a nivel de micro regiones para la intensidad promedio y el lapso promedio requerido para la adopción.

\section{Efectos a nivel individual}

La tabla 5 muestra los datos respectivos a las variables a utilizar en la prueba de las hipótesis 3 y 4 sobre efectos de la estructura de la red en la intensidad y prontitud de la adopción de las innovaciones. 
REDES- Revista hispana para el análisis de redes sociales

Vol.14,\#2, Junio 2006

http: // revista-redes.rediris.es

\begin{tabular}{|c|c|c|c|c|c|c|c|c|}
\hline Variable & Prom. & Desv.Est. & 1 & 2 & 3 & 4 & 5 & 6 \\
\hline 1. INTENSITY & 53,28 & 24,93 & - & & & & & \\
\hline 2. LAPSO & 1,13 & 2,25 & 0,00 & - & & & & \\
\hline 3. CENTRALID & 0,34 & 0,21 & $0,33^{\star \star}$ & 0,02 & - & & & \\
\hline 4. PROMOTOR & 3,69 & 1,10 & $0,27^{\star *}$ & $-0,11^{\star *}$ & $0,35^{\star *}$ & - & & \\
\hline 5. OTROSTEC & 2,34 & 1,07 & $-0,04$ & $-0,03$ & 0,40 ** & 0,01 & - & \\
\hline 6. OTROSMER & 2,58 & 0,87 & 0,05 & $-0,01$ & $0,45^{\star \star}$ & 0,02 & $0,21 * \star$ & - \\
\hline 7. OTROSPRO & 3,53 & 0,77 & $0,27^{\star \star}$ & $-0,05$ & 0,60 ** & 0,36 ** & 0,05 & $0,13^{*}$ \\
\hline 8. SIMMEL & 37,26 & 19,31 & $0,21^{\star *}$ & $-0,11$ & $0,47^{\star \star}$ & 0,08 & $0,48^{\star \star}$ & 0,59 ** \\
\hline 9. DISTANC & 26,25 & 38,66 & $-0,10$ & 0,09 & $-0,05$ & $-0,14^{*}$ & $-0,14^{\star}$ & $-0,10$ \\
\hline 10. CONSUMO & 32,91 & 37,37 & $-0,31^{\star \star}$ & $-0,01$ & $-0,24^{\star *}$ & 0,09 & 0,03 & $-0,37^{\star \star}$ \\
\hline 11. EDUCA & 2,96 & 1,02 & $0,09 *$ & $0,06^{*}$ & $-0,01$ & $-0,20$ ** & $0,15^{\star *}$ & 0,07 \\
\hline 12. PROPEXPE & 3,84 & 1,06 & $0,13^{\star \star}$ & $-0,04$ & $0,15^{\star \star}$ & $-0,04$ & $0,23^{\star \star}$ & $0,15^{\star \star}$ \\
\hline 13. PRODUCC & 3,74 & 0,92 & $0,11^{*}$ & 0,00 & $0,18^{\star *}$ & $0,12^{*}$ & 0,09 & 0,06 \\
\hline Variable & 7 & 8 & 9 & 10 & 11 & 12 & 13 & - \\
\hline 7. OTROSPRO & - & & & & & & & \\
\hline 8. SIMMEL & 0,10 & - & & & & & & \\
\hline 9. DISTANC & $-0,18^{\star \star}$ & 0,01 & - & & & & & \\
\hline 10. CONSUMO & $-0,20$ ** & $-0,39^{\star *}$ & 0,02 & - & & & & \\
\hline 11. EDUCA & $-0,09$ & $-0,01$ & $-0,04$ & 0,01 & - & & & \\
\hline 12. PROPEXPE & 0,03 & 0,07 & $-0,01$ & $-0,08$ & $0,30^{\star *}$ & - & & \\
\hline 13. PRODUCC & 0,08 & $0,12^{\star *}$ & $-0,00$ & $-0,15^{\star *}$ & 0,01 & $0,15^{\star *}$ & - & \\
\hline
\end{tabular}

Los coeficientes de correlación corresponden al test de Pearson excepto para las variables de ordinales (nos. $4,11,12$ y 13), para las que se indica el coeficiente del test Tau-B de Kendall. $* * p<.01 ; *<.05$

Tabla 5. Promedios, desviaciones estándar y correlaciones entre variables a nivel individual.

Los test de correlación muestran una asociación positiva y significativa entre muchas de las variables independientes y la intensidad de adopción. Según los mismos, los productores presentan un mayor grado de uso de la innovación cuando tienen una mayor centralidad del productor en la red, una mayor frecuencia de interacción con el promotor de la innovación y con otros productores, un mayor grado de vínculos simmelianos, un menor autoconsumo de su producción, mayores niveles educativo y de propensión a la experimentación, y cuando perciben que la innovación acarreará mayores incrementos en la producción. En cuanto al lapso requerido para la adopción, el mismo es significativamente menor para los productores que tienen una interacción más frecuente con el promotor de la innovación y se ve alargado levemente entre los productores con niveles de educación mayor. En todos los efectos son los que cabría esperar de acuerdo con la teoría, salvo en el caso de la educación y el lapso requerido para adoptar. En el presente caso, el efecto es pequeño pero significativo, lo que podría interpretarse como que un mayor nivel educativo en estas micro regiones implica que los 
REDES- Revista hispana para el análisis de redes sociales

Vol.14,\#2, Junio 2006

http://revista-redes.rediris.es

productores requieren algo más de tiempo y discusión para ser convencidos y/o convencerse a sí mismos antes de optar por la innovación.

Los coeficientes de correlación obtenidos son acordes con la mayor parte de lo previsto según las hipótesis 3 y 4 . En el caso de la intensidad, la interacción con los agentes del mercado no parece jugar mayor papel, salvo quizá cuando se trata de agentes fuertemente vinculados con el promotor de la innovación, como se ve reflejado por la correlación positiva y significativa del índice de vínculos simmelianos con la frecuencia de interacción con los agente del mercado. Por otro lado, la relación es contraria a lo estipulado en parte d) de la hipótesis 4, donde se partió del supuesto de que las comunidades en las micro regiones de análisis se comportarían de forma conservadora. Por el contrario, la evidencia muestra que una mayor interacción con otros productores conduce a una mayor intensidad de adopción. Esto podría reflejar también el hecho de que los promotores de la innovación en estas micro regiones se apoyan en técnicas grupales de comunicación/extensión, lo que queda reflejado en la asociación alta y significativa entre las frecuencia de interacción con el promotor y con los otros productores.

En el caso del lapso requerido para adoptar, aunque sólo uno de los coeficientes fue significativo, el signo de la asociación en casi todos los casos fue negativo (de nuevo, en el sentido previsto salvo en el caso de la interacción con otros productores, que una vez más parece favorecer la innovación). Sólo para el índice de centralidad se tuvo una asociación negativa, lo que podría sugerir que algunos elementos del conjunto de innovaciones podrían ser algo cuestionados culturalmente, razón por la cual los líderes de opinión tomarían algo más de tiempo en aceptarlos.

Este análisis también revela claramente que el indicador de centralidad utilizado está fuerte y significativamente correlacionado con todas las demás variables relativas a la interacción social (lo cual es esperable debido a la propia forma de calcular la centralidad), así como algunas de las variables sociodemográficas cuya asociación era menos obvia pero igualmente razonable (a mayor centralidad, menor autoconsumo, mayor propensión a la experimentación y mayores expectativas en torno a los beneficios de la innovación).

Los resultados anteriores fueron útiles también para seleccionar las variables relacionales a incluir como parte del modelo de regresión, a fin de evitar problemas de multicolinearidad. Se optó por utilizar dos versiones del modelo, incluyendo en una sólo el indicador de centralidad de grados del productor, y en la otra, las 
REDES- Revista hispana para el análisis de redes sociales

Vol.14,\#2, Junio 2006

http://revista-redes.rediris.es

variables relacionadas con la frecuencia de interacción con el promotor y con los otros productores, y el índice de presencia de vínculos simmelianos.

La tabla 6 muestra los resultados de los análisis comparativos de regresión Tobit, utilizando modelos con y sin variables relativas a la interacción social. El número de observaciones válidas se redujo de 360 a 296 por causa de datos perdidos para algunas de las variables consideradas en el modelo. En el modelo 1 ( $\sin$ variables relacionales), la variable más determinante de la intensidad de la adopción corresponde al indicador de las utilidades esperadas gracias a la innovación. En el modelo 2 se incluyó la centralidad de grado del productor como variable relacional (no se incluyeron otras en vista del alto grado de correlación de la misma con las otras variables relacionales consideradas). Dicha variable resultó ser la más determinante de la intensidad de adopción. Además, el grado de ajuste del modelo mejora marginalmente, en tanto que la relevancia de las otras variables consideradas mantiene el mismo patrón observado en el modelo 1. Finalmente, en el modelo 3 se incluyen como variables relacionales las frecuencias de interacción con el promotor y con otros productores, así como el índice de vínculos simmelianos. En este último caso el grado de ajuste del modelo se reduce sensiblemente, pero sus resultados nos permiten adentrarnos desagregar parcialmente el efecto causado en el modelo anterior por el índice de centralidad. Como se indicó en la metodología, los vínculos analizados en este trabajo tienen valor asignado de acuerdo con la frecuencia de la interacción. Por lo tanto una mayor centralidad en esta red significa un mayor número de vínculos frecuentes. El modelo 3 nos permite visualizar algo ya anunciado por los coeficientes de correlación: son los vínculos frecuentes con el promotor de la innovación, y con otros productores, los que realmente resultan determinantes. 
REDES- Revista hispana para el análisis de redes sociales

Vol.14,\#2, Junio 2006

http://revista-redes.rediris.es

\begin{tabular}{|c|c|c|c|}
\hline Variable & Modelo 1 & Modelo 2 & Modelo 3 \\
\hline Sigma (constante) & $\begin{array}{c}19.779 * * * \\
(.842)\end{array}$ & $\begin{array}{c}19.586 * * * \\
(.834)\end{array}$ & $\begin{array}{c}17.512 * * * \\
(.782)\end{array}$ \\
\hline CENTRALID & - & $\begin{array}{c}13.718^{* *} \\
(5.873)\end{array}$ & - \\
\hline PROMOTOR & - & - & $\begin{array}{c}5.720 * * * \\
(1.212)\end{array}$ \\
\hline OTROSPRO & - & - & $\begin{array}{c}3.045^{* *} \\
(1.501)\end{array}$ \\
\hline SIMMEL & - & - & $\begin{array}{c}.003 \\
(.060)\end{array}$ \\
\hline DISTANC & $\begin{array}{l}-.040 \\
(.033)\end{array}$ & $\begin{array}{l}-.039 \\
(.032)\end{array}$ & $\begin{array}{l}-.034 \\
(.030)\end{array}$ \\
\hline CONSUMO & $\begin{array}{c}-.119 * * * \\
(.032)\end{array}$ & $\begin{array}{c}-.108 * * * \\
(.032)\end{array}$ & $\begin{array}{c}-.146 * * * \\
(.032)\end{array}$ \\
\hline EDUCAC & $\begin{array}{c}3.808 * * * \\
(1.164)\end{array}$ & $\begin{array}{c}3.879 * * * \\
(1.153)\end{array}$ & $\begin{array}{c}3.246 * * * \\
(1.120)\end{array}$ \\
\hline PROPEXPE & $\begin{array}{c}4.971 * * * \\
(1.158)\end{array}$ & $\begin{array}{c}4.360 * * * \\
(1.177)\end{array}$ & $\begin{array}{c}1.811 \\
(1.154)\end{array}$ \\
\hline PRODUCC & $\begin{array}{c}8.297 * * * \\
(1.021)\end{array}$ & $\begin{array}{c}7.508 * * * \\
(1.066)\end{array}$ & $\begin{array}{c}3.918^{* * *} \\
(1.161)\end{array}$ \\
\hline \multicolumn{4}{|l|}{ Fit measures: } \\
\hline Log Likelihood function & -1215.381 & -1212.680 & -1074.741 \\
\hline ANOVA based fit & .355 & .360 & .273 \\
\hline DECOMP based fit & .267 & .273 & .259 \\
\hline
\end{tabular}

Error estándar entre paréntesis; $* * * p<.01 ; * * p<.05 ; * p<.10$

Tabla 6. Coeficientes Tobit para predecir la intensidad de adopción individual $(n=296)$.

El modelo 3 también nos deja ver cómo algunas variables que parecían clave pierden completamente su poder de predicción con una pequeña variación en la especificación del modelo. Tal es el caso de la propensión a la experimentación (la actitud hacia el riesgo). En suma, esta sencilla comparación de modelos permite apuntar evidencias constructivas para acercar tradiciones: no son única ni primordialmente las variables económicas las que a la larga determinan el comportamiento individual ante la innovación, pero tampoco se trata de un proceso de contagio social en el que no median las valoraciones individuales sobre costos y beneficios. Se obtiene una imagen más ponderada del aporte de ambos tipos de factores cuando se les combina en un modelo econométrico, algo que resulta facilitado mediante los diversos parámetros estructurales que se pueden generar a través del ARS, y sin que los modelos vean reducida su capacidad de explicativa. 
REDES- Revista hispana para el análisis de redes sociales

Vol.14,\#2, Junio 2006

http: // revista-redes.rediris.es

Por último, con respecto a la hipótesis 5, el coeficiente de correlación de Pearson obtenido a través del test de correlación QAP entre las matrices de similitudes de Jaccard y de diferencias entre los niveles de adopción de cada díada, fue - 0.073 (** $p$ <.05). Como se aprecia, el nivel de asociación entre ambas matrices es bajo pero significativo y negativo, de forma que a mayor similitud entre dos productores al respecto de su estructura de vínculos, menor diferencia existirá en sus niveles de adopción. Para confirmar estos datos, el mismo test se realizó a lo interno de cada micro región (datos no mostrados): el coeficiente de regresión fue negativo y significativo en 8 de ellas, negativo pero no significativo en otras tres, y positivo y no significativo en tan sólo una micro región. De esta forma, la evidencia obtenida brinda soporte a la existencia de una débil pero consistente asociación entre la equivalencia estructural y la intensidad de adopción, lo que equivale a hablar de una cierta tendencia, entre los productores consultados, a competir y/o imitar a sus referentes ${ }^{11}$.

\section{Discusión}

Este trabajo presenta evidencia sobre procesos de influencia social y competencia que estarían incidiendo en las decisiones de los pequeños productores con respecto a su grado de adopción de innovaciones. La influencia social (i.e., las presiones para cumplir con las normas y estándares aceptados socialmente) fue evidenciada mediante las pruebas de hipótesis relativas a los efectos de la cohesión sobre los niveles de adopción, tanto a nivel de red como de díadas (hipótesis 1,3 y 4e). En términos generales, a más intensa la actividad de la red en que se participa, y la participación individual del propio productor, menores son las probabilidades de comportarse de una forma distinta a la de sus pares. En las micro regiones estudiadas, que se muestran receptivas a las innovaciones promovidas, la presión por actuar conforme con lo esperado socialmente actúa a favor de la innovación, induciéndola. Por otro lado la competencia con los referentes sociales, un proceso según el cual los productores se verían movidos hacia la innovación una vez que sus semejantes lo hayan hecho (aquellos con patrones semejantes de vinculación social, sin que se requiera que entre ellos medie vínculo alguno), también fue

\footnotetext{
${ }^{11}$ Se optó por no incluir la equivalencia estructural en el modelo econométrico debido a que este efecto debería incidir básicamente a nivel local o, a lo sumo, al nivel de actividad (i.e., los grupos de referencia de estos pequeños productores, de cara a la innovación, se limitarían a esas esferas). La forma de incluir la equivalencia estructural como una variable individual sujeta de análisis econométrico sería, como se indicó en la metodología, generando grupos con niveles de equivalencia similar mediante agrupamiento jerárquico, que luego entrarían en el modelo como dummies. Pero tal procedimiento nos conduciría a un análisis detallado, ya sea por actividad y/o por localidad, lo que no es el cometido de este informe.
} 
REDES- Revista hispana para el análisis de redes sociales

Vol.14,\#2, Junio 2006

http: //revista-redes.rediris.es

evidenciada mediante la evidencia obtenida sobre los efectos de la equivalencia estructura (hipótesis 5).

Las medidas de cohesión utilizadas en el presente estudio deben, no obstante, tomarse con alguna cautela, dado que no se derivan de los vínculos existentes en una red social en sentido estricto. Como se indicó en la metodología, los datos relacionales obtenidos para el presente estudio permiten sacar provecho de las herramientas del ARS para su análisis, pero describen ante todo la afiliación de los productores con ciertos agentes (individuales o categóricos) de cambio ${ }^{12}$. Aún y cuando, entre dichos "agentes", se incluyó a los otros productores, las interacciones individuales entre productores no fueron abordadas.

En este sentido, las medidas de densidad a nivel de red nos dan tan sólo una aproximación gruesa a la cohesión que podría obtenerse al considerar el conjunto completo de pares (y de otros actores aquí tratados como categorías) con quienes cada productor interactúa. Podría argüirse que, al estimar los datos de esta forma, la contribución de los agentes externos aparece sobre-estimada en las medidas de cohesión. En respuesta cabe argumentar que este tipo de tratamiento es útil también para abarcar las diferencias cualitativas entre vínculos horizontales (entre pares) y verticales (con autoridades o agentes externos, más asimétricos). En todo caso, y a pesar de estas deficiencias - que estarían introduciendo en todo caso un sesgo sistemático-, la evidencia obtenida permitió dar soporte a las hipótesis que predicen efectos positivos de la cohesión, la prominencia (centralidad) individual y la competencia por status, sobre el grado de participación de los pequeños productores en los procesos de innovación.

A pesar de que el tipo de información utilizada no es óptima para un análisis en profundidad de las interacciones sociales, es importante destacar a la vez que los procedimientos descritos en este trabajo dan una buena idea de los muchos aspectos de las relaciones sociales que pueden explorarse aún con sets de datos derivados de trabajos econométricos convencionales, con la simple adición de unas breves preguntas sobre las interacciones con distintos actores en los instrumentos de consulta.

\footnotetext{
12 En este estudio se les designó "Agentes de Influencia Social" puesto que el más tradicional "Agentes de Cambio" se circunscribe casi siempre a los agentes técnicos externos, en tanto que el presente análisis quiere dar cuenta, con ese título, de la serie de actores presentes en cada microrregión, tanto externos como internos, que ejercen algún tipo de influencia sobre la toma de decisiones de los productores.
} 
REDES- Revista hispana para el análisis de redes sociales

Vol.14,\#2, Junio 2006

http: // revista-redes.rediris.es

En particular, el enfoque utilizado es sumamente útil para analizar la interacción del productor con otros actores externos, elemento central de la hipótesis 4 y sobre el que la evidencia provista por este estudio fue bastante clara en cuanto a su papel central como determinante del proceso de adopción. Este tipo de relación está un tanto marginada en la literatura sobre ARS e innovación, y resulta de particular importancia en escenarios de desarrollo, donde una diversidad de agentes se aproxima a propósito y de forma recurrente a los pequeños productores, con el objeto de promover, poner en práctica, desarrollar conjuntamente y/o brindar soporte a la implementación de innovaciones institucionales, organizacionales y técnicas. Estos agentes externos utilizan, para tales efectos, distintos mecanismos de persuasión que tienen enorme efecto sobre las tasas de adopción. Por ello es fundamental comprender mejor tales interacciones y la forma en que las mismas pueden mejorarse con el propósito de hacer de la innovación un proceso más efectivo, equitativo y empoderador.

De cara al futuro es necesario realizar estudios en esta línea pero que profundicen en las interacciones a lo interno de las comunidades, lo cual permitiría explorar en mayor detalle los distintos roles ejercidos por distintos productores (tal como el de los líderes de opinión, de vieja data en los estudios sobre difusión de innovaciones agrícolas). Para ello se requiere utilizar nuevos enfoques de cara al muestreo, utilizando por ejemplo la técnica de bola de nieve, o en concentrar los esfuerzos en pequeños estudios de caso locales tratados en profundidad. Los estudios de caso permitirían abordar otro aspecto importante, cual es el de la multiplicidad de relaciones (redes) que ligan a un mismo conjunto de actores (p.ej., relaciones de intercambio de productos, de información, de acceso a crédito, etc.), todas las cuales pueden ejercer distintas influencias sobre el proceso de innovación. Se debe, por último, detallar más en los agentes específicos, sus relaciones con cada productor individual, y los mecanismos de persuasión utilizados, como paso para comprender mejor los procesos sociales que afectan el cambio de actitudes que sirve de antesala a la adopción de innovaciones.

\section{Bibliografía}

Abadi Ghadim, A. K.; Pannell, D. J.; Burton, M. P. (2005). Risk, uncertainty, and learning in adoption of a crop innovation. Agricultural Economics 33: 1-9.

Bandiera, O.; Rasul, I. (2006). Social Networks and Technology Adoption in Northern Mozambique. Economic Journal 116 (514): 869-902.

Bebbington, A. (1999). Capitals and Capabilities: A Framework for Analyzing Peasant Viability, Rural Livelihoods and Poverty. World Development 27: 2021-2044. 
REDES- Revista hispana para el análisis de redes sociales

Vol.14,\#2, Junio 2006

http://revista-redes.rediris.es

Becker, M. H. (1970). Sociometric Location and Innovativeness: Reformulation and Extension of the Diffusion Model. American Sociological Review 35 (2): 267-282.

Birkhaeuser, D.; Evenson, R. E.; Feder, G. (1991). The Economic Impact of Agricultural Extension: A Review. Economic Development and Cultural Change 39: 507-521.

Boahene, K. (1995). Innovation Adoption as a Socio-Economic Process: The Case of Ghanaian Cocoa Industry. Ph.D. Dissertation. Utrecht, The Netherlands: Utrecht University.

Boahene, K.; Snijders, T. A. B.; Folmer, H. (1999). An Integrated Socioeconomic Analysis of Innovation Adoption: The Case of Hybrid Cocoa in Ghana. Journal of Policy Modelling 21 (2): $167-184$

Borgatti, S. P. (2005). Centrality and network flow. Social Networks 27: 55-71.

Borgatti, S. P.; Everett, M. G.; Freeman, L. C. 2002. Ucinet for Windows: Software for Social Network Analysis. Harvard: Analytic Technologies.

Burt, R. S. (1980). Innovation as a Structural Interest: Rethinking the Impact of Network Position on Innovation Adoption. Social Networks 2: 327-355.

Burt, R. S. (1987). Social Contagion and Innovation: Cohesion versus structural equivalence. American Journal of Sociology 92: 1287-1335.

Burt, R. S. (1992). Structural Holes: The Social Structure of Competition. Cambridge: Harvard University Press.

Burt, R. S. (2005). Brokerage and Closure: An Introduction to Social Capital. Oxford: Oxford University Press.

Carey, H. A. (1999). Communication in Extension. A teaching and learning guide. Rome: FAO.

Caviglia-Harris, J. L. (2003). Sustainable Agricultural Practices in Rondonia, Brazil: Do Local Farmer Organizations Affect Adoption Rates. Economic Development and Cultural Change 52: $23-49$

Coleman, L. (1951). Differential Contact with Extension Work in a New York Rural Community. Rural Sociology 16: 207-216.

Conley, T. G.; Udry, C. R. (2004). Learning About a New Technology: Pineapple in Ghana. Discussion Paper No. 817. Yale University - Economic Growth Center.

Conley, T. G.; Udry, C. R. (2001). Social learning through networks: the adoption of new agricultural technologies in Ghana. American Journal of Agricultural Economics 83 (3): 668673.

Copp, J. H. (1958). Toward generalization in farm practice research. Rural Sociology 23: 103-111.

Dagenne, A.; Forsé, M. (1999). Introducing Social Networks. London, Sage Publications.

Dinar, A.; Karagiannis, G.; Tzouvelekas, V. (2007). Evaluating the impact of agricultural extension on farms' performance in Crete: a nonneutral stochastic frontier approach. Agricultural Economics 36: 135-146.

Doss, C. R. (2006). Analyzing technology adoption using microstudies: limitations, challenges and opportunities for improvement. Agricultural Economics 34: 207-219.

Durston, J. (2002). El capital social campesino en la gestión del desarrollo rural. Diadas, equipos, puentes y escaleras. Santiago de Chile: CEPAL. 
REDES- Revista hispana para el análisis de redes sociales

Vol.14,\#2, Junio 2006

http: //revista-redes.rediris.es

Erickson, B. H. (1988). The relational basis of attitudes. In: Social structures: a network approach. Wellman, B.; Berkowitz, S. D. (eds.). New York: Cambridge University Press.

Everett, M. G.; Borgatti, S. P. (2005). Extending Centrality. In: Models and Methods in Social Network Analysis. Carrington, P.J; Scott, J \& S. Wasserman (eds). New York: Cambridge University Press.

Fafchamps, M. (2007). The formation of risk sharing networks. Journal of Development Economics 83: 326-350.

Fafchamps, M. (2006). Development and Social Capital. Journal of Development Studies 42: $1180-1198$.

Fagerber, J., Mowery, D. C., R. R. Nelson. (2004). The Oxford Handbook of Innovation. Oxford, Oxford University Press.

Feder, G.; Lau, L. J.; Slade, R. H. (1987). Does Agricultural Extension Pay? The Training and Visit System in Northwest India. American Journal of Agricultural Economics 69: 677-686.

Feder, G.; Savastano, S. (2006). The Role of Opinion Leaders in the Diffusion of New Knowledge: The Case of Integrated Pest Management. World Development 34 (7): 12871300.

Foster, D.; Rosenzweig, M. (1995). Learning by Doing and Learning from Others: Human Capital and Technical Change in Agriculture. Journal of Political Economy 103: 1176-1209.

Frambach, R. T. (1993). An Integrated Model of Organizational Adoption and Diffusion of Innovations. European Journal of Marketing 27 (5): 22-41.

Friedkin, N. E. (1984). Structural Cohesion and Equivalence Explanations of Social Homogeneity. Sociological Methods and Research 12: 234-261.

Friedkin, N. E. (1999). Social Influence Networks and Opinion Change. Advances in Group Processes 16: 1-29.

Friedkin, N. E. and E. C. Johnssen (1997). Social positions in influence networks. Social Networks 19: 209-222.

Glendinning, A.; Mahapatra, A.; Mitchell, C. P. (2001). Modes of Communication and Effectiveness of Agroforestry Extension in Eastern India. Human Ecology 29: 283-305.

Goyal, S. (2007). Connections: An introduction to the economics of networks. Princeton N.J .: Princeton University Press.

Granovetter, M. (1982). The strength of weak ties: A network theory revisited. In: Social Structure and Network Analysis. Marsden, P. V.; Lin, N. (eds.). Newbury Park, CA.: Sage.

Granovetter, M. (1973). The strength of weak ties. American Journal of Sociology 78: 1360-1380.

Granovetter, M. (2005). The Impact of Social Structure on Economic Outcomes. Journal of Economic Perspectives 19: 33-50.

Greene, W. H. (1990). Econometric Analysis. New York: McMillan.

Griliches, Z. (1957). Hybrid Corn: An Exploration in the Economics of Technological Change. Econometrica 25: 501-522.

Grootaert, C. and T. van Bastelaer (2008). The role of social capital in development: An empirical assessment. Cambridge, MA: Cambridge University Press. 
REDES- Revista hispana para el análisis de redes sociales

Vol.14,\#2, Junio 2006

http: // revista-redes.rediris.es

Hanneman, R. A., M. Riddle (2005). Introduction to social network methods. Riverside, CA: University of California, Riverside (published in digital form at http://faculty.ucr.edu/ hanneman/)

Hogset, H. (2005). Social Networks and Rural Development: Theory and Applications in the Kenyan Highlands. Ph.D. Dissertation. Ithaca, New Cork: Cornell University

Hogset, H. (2005). Social Networks and Technology Adoption. BASIS Policy Brief No. 6. Basis Collaborative Research Support Program. Ithaca, New York: Cornell University.

Hussain, S.S.; Byerlee, D.; Heisey, P.W. (1994). Impacts of training and visit extension system on farmers' knowledge and adoption of technology: evidence from Pakistan. Agricultural Economics 10: 39-47.

Isham, J. (2002). The effect of Social Capital on Technology Adoption: Evidence from Rural Tanzania. Journal of African Economies 11: 39-60.

Katungi, E. M., Edmeades, S., M. Smale (2008). Gender, Social Capital and Information Exchange in Rural Uganda. Journal of International Development 20: 32-52.

Kohler, H-P., Behrman, J. R.; Watkins, S. C. (2007). Social Networks and HIV/AIDS Risk Perceptions. Demography 44: 1-33.

Krackhardt, D. (1987). QAP Partialling as a test of spuriousness. Social Networks 9: 171186.

Krackhardt, D. (1988). Predicting with networks: Nonparametric multiple regression analysis of dyadic data. Social Networks 10: 359-381.

Krackhard, D. (1998). Simmelian Ties: Super Strong and Sticky. In: Power and Influence in Organizations. R. Kramer \& M. Neale (eds.). Thousand Oaks, California, Sage.

Krackhardt, D. (1999). The ties that torture: Simmelian tie analysis in organizations. Research in the Sociology of Organizations 16: 183-210.

Krackhardt, D.; Kilduff, M. (2002). Structure, culture and Simmelian Ties in Entrepreneurial Firms. Social Networks 24: 279-290.

Kranton, Rachel E. y Minehart, D. (2001). A Theory of Buyer-Seller Networks. American Economic Review 91 (3): 485-508.

Levin, D. Z.; Cross, R. (2004). The Strength of Weak Ties you can Trust: The Mediating Role of Trust in Effective Knowledge Transfer. Management Science 50: 1477-1490.

Lin, N.; Burt, R. S. (1975). Differential Effects of Information Channels in the Process of Information Diffusion. Social Forces 54 (1): 256-274.

Liu, W. T.; Duff, R. W. (1972). The Strength in Weak Ties. The Public Opinion Quarterly 36: 361-366.

Manski, C. F. (2000). Economic Analysis of Social Interactions. Journal of Economic Perspectives 14: 115-136.

Marsden, P. V.; Friedkin, N. E. (1994). Network Studies of Social Influence. In: Advances in Social Network Analysis. Research in the Social and Behavioral Sciences. Wasserman, S.; Galaskiewicz, J. (eds.). London: Sage.

McPherson, M.; Smith-Lovin, L.; Cook, J. M. (2001). Birds of a Feather: Homophily in Social Networks. Annual Review of Sociology 27: 415-444. 
REDES- Revista hispana para el análisis de redes sociales

Vol.14,\#2, Junio 2006

http: // revista-redes. rediris.es

Michaelson, A.; Contractor, N. S. (1992). Structural Position and Perceived Similarity. Social Psychology Quarterly 55:

Mizruchi, M. S. (1993). Cohesion, equivalence and similarity of behavior: a theoretical and empirical assessment. Social Networks 15: 275-307.

Monge, P. R.; Contractor, N. S. (2003). Theories of Communication Networks. New York: Oxford University Press.

Moser, C. M.; Barrett, C. B. (2006). The Complex Dynamics of Smallholder Technology Adoption: The Case of SRI in Madagascar. Agricultural Economics 35: 373-388

Moxley, R. L.; Lang, K. B. (2006). The importance of social context influences on new farm technology sustainability: community and sub-community characteristics in Jamaica. Technology in Society 28: 393-406.

Munshi, K. (2005). Social Learning in a Heterogeneous Population: Technology Diffusion in the Indian Green Revolution. Journal of Development Economics 73 (1): 185-215.

Nelson, R.R., and K. Nelson (2002). Technology, institutions and innovation systems. Research Policy 31: 265-272.

Newell, S.; Swan, J. (1995). Professional associations as important mediators of the innovation process. Science Communication 16: 371-387.

Nyblom, J.; Borgatti, S.; Roslakka, J.; Salo, M. A. (2003). Statistical analysis of network data - an application to diffusion of innovation. Social Networks 25: 175-195.

Obstfeld, D. (2005). Social Networks, the Tertius I ungens Orientation, and Involvement in Innovation. Administrative Science Quarterly 50: 100-130.

Okten, Cagla y Osili, U. O. (2004). Social Networks and Credit Access in Indonesia. World Development 32 (7): 1225-1246.

Opare, K. D. (1977). The role of Agricultural Extension in the Adoption of Innovations by Cocoa Growers in Ghana. Rural Sociology 42: 72-82.

Polgar, S.; Dunphy, H.; Cox, B. (1963). Diffusion and Farming Advice: A Test of Some Current Notions. Social Forces 42: 104-111.

Pomp, M.; Burger, K. (1995). Innovation and Imitation: Adoption of Cocoa by Indonesian Smallholders. World Development 23 (3): 423-431.

Reagans, R.; McEvily, B. (2003). Network Structure and Knowledge Transfer: The Effects of Cohesion and Range. Administrative Science Quarterly 48: 240-267.

Rogers, E. M. (2003). Diffusion of Innovations. 5 ed. New York: Free Press.

Rogers, E. M.; Beal, G. M. (1958). The Importance of Personal Influence in the Adoption of Technical Changes. Social Forces 36 (4): 329-335.

Ruttan, V. W. (1996). What happened to technology adoption-diffusion research? Sociologia Ruralis 36: 51-73.

Ryan, B.; Gross, N. C. (1943). The diffusion of hybrid seed corn in two lowa communities. Rural Sociology 8: 15-24.

Serageldin, I., and P. Dasgupta, eds. (2001). Social capital: A multifaceted perspective. Washington, DC: World Bank. 
REDES- Revista hispana para el análisis de redes sociales

Vol.14,\#2, Junio 2006

http: // revista-redes.rediris.es

Sparrowe, R. T.; Liden, R. C. (2005). Two Routes to Influence: Integrating Leader-Member Exchange and Social Network Perspectives. Administrative Science Quarterly 50: 505-535.

Strang, D.; Soule, S. (1998). Diffusion in Organizations and Social Movements: From Hybrid Corn to Poison Pills. Annual Review of Sociology 24: 265-290.

Strauss, J.; Barbosa, M.; Teixeira, S.; Thomas, D.; Gomes Junior, R. (1991). Role of education and extension in the adoption of technology: a study of upland rice and soybean farmers in Central-West Brazil. Agricultural Economics 5: 341-359.

Tuomi, I. (2002). Networks of Innovation. Change and meaning in the Age of the Internet. New York: Oxford University Press.

Valente, T. W. (1996). Social network thresholds in the diffusion of innovations. Social Networks 18: 69-89.

Valente, T. W. (2005). Network Models and Methods for Studying the Diffusion of Innovations. In: Models and Methods in Social Network Analysis. Carrington, P.J; Scott, J \& S. Wasserman (eds). New York: Cambridge University Press.

Van den Broeck, Katleen y Dercon, S. (2007). Social Interactions in Growing Bananas: Evidence from a Tanzanian Village. CSAE Working Paper 2007-05.

Van den Bulte, C.; Lillien, G. L. (2001). Medical innovation revisited: Social contagion versus marketing effort. American Journal of Sociology 106: 1409-1435.

Wasserman, S.; Faust, K. (1994). Social Network Analysis. Methods and Applications. New York: Cambridge University Press.

Wejnert, B. (2002). Integrating Models of Diffusion of Innovations: A Conceptual Framework. Annual Review of Sociology 28: 297-326.

Wilkening, E. A. (1956). Roles of communicating agents in technological change in agriculture. Social Forces 34: 361-367.

Woolcock, M.; Narayan, D. (2000). Social Capital: Implications for Development Theory, Research and Policy. The World Bank Research Observer 15: 225-249.

Yamauchi, F. (2007). Social learning, neighbourhood effects and investment in human capital: Evidence from Green-Revolution India. Journal of Development Economics 83: 3762. 